\title{
Comparative transcriptome analysis provides new insights into the molecular regulatory mechanism of adventitious root formation in ramie (Boehmeria nivea L.)
}

Kunmei Chen ( $\nabla$ chenkunmei@caas.cn )

IBFC,CAAS

Bing Guo

IBFC, CAAS

Chunming Yu

Chinese Academy of Agricultural Sciences Feed Research Institute

Ping Chen

IBFC, CAAS

Jikang Chen

Oil Crops Research Institute Chinese Academy of Agricultural Sciences

Gang Gao

Oil Crops Research Institute Chinese Academy of Agricultural Sciences

Xiaofei Wang

Oil Crops Research Institute Chinese Academy of Agricultural Sciences

Aiguo Zhu

IBFC, CAAS

Research article

Keywords: Ramie (Boehmeria nivea L.), adventitious roots, hydroponics, RNA-Seq, transcriptomic divergence

Posted Date: April 30th, 2020

DOl: https://doi.org/10.21203/rs.3.rs-23123/v1

License: (9) (1) This work is licensed under a Creative Commons Attribution 4.0 International License. Read Full License 
Version of Record: A version of this preprint was published at Plants on January 15th, 2021. See the published version at https://doi.org/10.3390/plants10010160. 


\section{Abstract}

Background: The occurrence of adventitious roots is necessary for the survival of cuttings. In this study, comparative transcriptome analysis between two ramie (Boehmeria nivea L.) varieties with different adventitious root (AR) patterns, was performed by RNA-Seq under water culture (treatment, $T$ ) and nonwater culture conditions (control, $\mathrm{CK}$ ) to reveal the regulatory mechanism of rooting.

Results: Characterization of the cuttings of two ramie cultivars, Zhongzhu No 2 (Z2) and Huazhu No 4 $(\mathrm{H} 4)$, indicated that $\mathrm{Z} 2$ had high adventitious rooting rate but $\mathrm{H} 4$ had low rooting rate. Twelve cDNA libraries of the two varieties were constructed, and a total of 26723 genes were identified. In non-water culture condition, the number of the distinctive genes in $\mathrm{H} 4$ was 2.7 times of that in $\mathrm{Z2}$, while in water culture condition, the number of the distinctive genes in $\mathrm{Z} 2$ was nearly 2 times of that in $\mathrm{H} 4$. A total of 4411 and 5195 DEGs were identified in the comparison of H4CK vs. H4T and Z2CK vs. Z2T, respectively. After water culture, more DEGs were up-regulated in Z2, but more DEGs were down-regulated in H4. Gene Ontology (GO) Gene functional analysis of the DEGs indicated that polysaccharide metabolic process, carbohydrate metabolic process, cellular carbohydrate metabolic process, cell wall macromolecule metabolic process, and photosystem $\mathrm{GO}$ terms were distinctive significantly enriched in $\mathrm{H} 4$. Simultaneously, Kyoto Encyclopedia of Genes and Genomes (KEGG) analysis showed that photosynthesis, photosynthesis-antenna proteins and starch and sucrose metabolism pathways were identified in H4. Moreover, KEGG analysis showed that jasmonic acid (JA) can interact with ethylene to regulate the occurrence and number of $A R$ in $Z 2$.

\section{Conclusions:}

This study reveals the transcriptomic divergence of two ramie varieties with high and low adventitious rooting rates, and provides insights into the molecular regulatory mechanism of adventitious root formation in ramie.

\section{Background}

The rooting ability of clones is the key factor to determine the success of propagation of cuttings, and it is also the main criterion to distinguish the adaptability and resistance of clones. Adventitious root (AR) is the root of cuttage plants, which is an important organ of water and inorganic salt absorption and assimilate storage, and plays an important role in stress resistance [1, 2]. The occurrence of AR enlarges the root system of plants and enables plants and cells to regenerate. In most plants, AR originates from flood conditions. When plants suffer from hypoxia or other stress, AR can significantly improve the survival ability of plants [3]. Therefore, in-depth understanding of the mechanism of AR is helpful to improve the survival rate of cuttings and the survival ability of plants under stress.

Ramie (Boehmeria nivea L.), which has a history of over 4,000 years in China, is currently mainly planted in China, India, and other Southeast Asian and Pacific Rim countries. It can be used as an excellent fibre crop, a forage plant, a soil and water conservation plant, and as an ideal crop for cadmium pollution 
remediation [4, 5]. Ramie is a highly heterozygous crop [6], thus, it is impossible to maintain the fine quality of ramie by seed propagation, but asexual propagation can solve the problem well. Cutting propagation is faster than seed reproduction, and can maintain the genetic stability of cultivars, which make it being a popular way of propagation in potato, strawberry, grape, kiwifruit, chestnut and ramie. With the development and utilization of ramie, demand of ramie raw materials is increasing. Asexual propagation can rapidly propagate a large number of ramie seedlings to meet the needs of production.

AR formation (ARF) is of utmost interest since it consists of the development of root tissues from nonroot and non-meristematic tissues. The formation and development of $A R$ are regulated by various biological processes, and its molecular regulative mechanism is complex. At present, it has been found that plant hormones play an important role in ARF. Indole-3-butyric acid can induce ARF in many plants such as Pinus thunbergii, Camellia sinensis, and Sterculia foetida [7-9]. In cucumber, abscisic acid can induce adventitious rooting of its explants under drought stress [10]. In tomato, ethylene plays a positive role in ARF and a negative role in lateral root formation with modulation of auxin transport as a central point of ethylene-auxin crosstalk [11]. In petunia, ethylene plays as sitmulator and auxin plays as regulator during ARF of petunia cutting [12]. Auxin acts as a central player in the hormone cross-talks that control adventitious rooting [13]. In hybrid aspen and Arabidopsis, gibberellin can inhibit adventitious rooting by affecting auxin transport [14]. In Morinda citrifolia and apple, cytokinin can inhibite AR primordia formation and AR growth [15, 16]. In Arabidopsis and pea, strigolactone suppresses adventitious rooting but promote for $\operatorname{ARF}$ in rice $[17,18]$. However, in ramie, whether plant hormones affect the AR in the cuttings is unknown. The molecular mechanism underlying ARF in ramie cutting propagation is still elusive.

RNA-Seq and comparative comics have been popularly used to explore pathways during AR formation and development [19-21]. Ramie cultivar Zhongzhu No2 (Z2) and Huazhu No4 (H4) show distinct rooting rate by cutting propagation in water culture. This study aims to preliminarily analyze the molecular mechanism of ARF in ramie cuttings by comparing the transcriptome information between Z2 and $\mathrm{H} 4$ performed by RNA-Seq, and hopes to identify the key pathways that involve in ARF in ramie. This study will be helpful to improve the survival rate of ramie cuttings.

\section{Methods}

\section{Ethics statement}

The ramie varieties used in this study were planted in our scientific research field, which is owned by our institution. Therefore, no specific permissions were required for using these plant materials.

\section{Plant Growth And Sampling}

Two ramie cultivars, Zhongzhu No $2(\mathrm{Z} 2)$ and Huazhu No $4(\mathrm{H} 4)$, were used in this study. The ramie plants grew in a greenhouse at $25 \pm 1{ }^{\circ} \mathrm{C}$ with a $12 \mathrm{~h}$ photoperiod, $75 \% \pm 1 \mathrm{RH}$, under light intensity of 
5000 lux. Shoots of $10-15 \mathrm{~cm}$ in length were collected and then inserted into conical flasks with tapwater, one shoot in one flask, and there were about $4-5 \mathrm{~cm}$ of the stem were submerged in the water. The tap-water was renewed every two days. The root primordia formation (RAF) was monitored every day. Ten days after water culture, stems with root primordium were collected as treatment samples (T). Stems without water treatment were collected as control samples (CK). All samples were immediately frozen in liquid nitrogen, and stored at $-80^{\circ} \mathrm{C}$. Three replicates were set for $\mathrm{CK}$ and $\mathrm{T}$ samples, respectively, and each replicate contained five individual plant.

\section{Rna Isolation, Library Preparation And Sequencing}

Total RNA was extracted from the samples with TRIzol Reagent (Invitrogen, Carlsbad, CA, USA) and purified with Plant RNA Purification regent (Invitrogen, Carlsbad, CA, USA) according to the manufacturer's protocol. The concentration and purity of total RNA was determined using NanoDrop2000 spectrophotometer (Thermo Fisher Scientific, Waltham, MA, USA), and RNA integrity was examined by Agilent2100 bioanalyzer (Agilent, Santa Clara, CA, USA).

For transcript sequencing, 12 libraries (Z2CK1, Z2CK2, Z2CK3, Z2T1, Z2T2, Z2T3, H4CK1, H4CK2, H4CK3, $\mathrm{H} 4 \mathrm{~T} 1, \mathrm{H} 4 \mathrm{~T} 2$ and H4T3) were constructed using $5 \mu \mathrm{g}$ of total RNA. Briefly, poly (A) mRNA was extracted from the total RNA sample using Oligo (dT) magnetic beads. mRNA was fragmented into 200-500 nt

pieces by adding a fragmentation buffer. First-strand cDNA was synthesized using SuperScript II reverse transcriptase (Life Technologies, Inc.) and random hexamer primers. After generation of second-strand cDNA, the double-strand cDNA was end-repaired, and a single ' $A$ ' base and indexed adapters were ligated to the fragments. The cDNA library was constructed with the Illumina Paired End Sample Prep kit (Illumina, USA), quantified by TBS380 (Picogreen, Invitrogen, USA) and was then sequenced on the Illumina HiSeq ${ }^{\mathrm{TM}} 2500$ ( $2 \times 150$ bp read length) platform. Illumina sequencing was performed at Majorbio Bio-pharm Technology Co. Ltd, Shanghai, China (http://www.majorbio.com/). Illumina sequencing data was deposited in the NCBI GEO database with accession number GSE116063.

\section{Data Filtering And Mapping}

Adapter sequences, low-quality reads $(\mathrm{Q}<20)$, extreme short sequences, and reads with unknown nucleotides larger than $5 \%$ were removed to obtain high-quality reads. The remaining reads were classified as clean reads. The clean reads were mapped to the ramie reference genome (data unpublished) using HISAT2 (v2.1.0, http://www.ccb.jhu.edu/software/hisat).

\section{Identification Of Differentially Expressed Genes (degs)}

The gene expression levels of the mapped reads were represented by the expected number of Fragments Per Kilobase of transcript sequence per Millions base pairs sequenced (FPKM), which was calculated by 
RSEM (v1.2.8, http://deweylab.biostat.wisc.edu/rsem/rsem-calculate-expression.html). DEGs were identified using the DESeq2 R package according to the method described by [22]. The resulting $p$-values were adjusted using the Benjamini and Hochberg's approach for controlling the false discovery rate. Genes were designated as differentially expressed when the adjusted $p$-value was $<0.001$ and estimated absolute log2 (fold change) $\geq 2$.

\section{Gene Ontology (go) And Pathway Enrichment Analysis Of Degs}

GO enrichment analysis of DEGs was performed using the cluster Profiler R pachage. GO terms with an adjusted $p$-value $<0.05$ were considered to be significantly enriched, and were categorized into three types of functional classification, namely cellular component, molecular function and biological process. Pathway analysis was performed using the Kyoto Encyclopedia of Genes and Genemes (KEGG) web server (https://www.kegg.jp/), and the enrichment analysis was conducted by the cluster Profiler R pachage. Pathways with an adjusted $p$-value $<0.05$ were considered to be significantly enriched.

\section{Quantitative Pcr (qpcr) Validation Of Degs}

Total RNA was reverse transcribed using a PrimeScript RT perfect real-time reagent kit (Takara, Japan), and then the CDNA was diluted four times. The qPCR analysis was performed using the special primers showed in Additional file 1: sheet1, according to the method of Chen et al. [23]. 18S rRNA gene was used as the internal control to normalize q-PCR data. Samples without water culture were set as control, and the relative expression of target genes of samples with water culture for different days was calculated using the $2^{-\triangle \Delta C t}$ method [24].

\section{Results}

\section{Adventitious root formation of two ramie cultivars}

In order to study the rooting rate of $\mathrm{Z} 2$ and $\mathrm{H} 4$, root primordia formation in water culture condition were monitored. Five days after water culture, white root primordia of $\mathrm{Z} 2$ and $\mathrm{H} 4$ emerged from the stem epidermis section in the water, and more root primordia were observed on Z2 than that on $\mathrm{H} 4$. At the seventh and the tenth day, there were significantly more root primordia on Z2 than that on H4 (Fig. 1).

\section{Transcriptome Sequencing Analysis Of Twelve Cdna Libraries}

In order to study the molecular mechanism of rooting difference between Z2 and H4, RNA-Seq analysis was performed on stems with root primordium induced by tap-water (treatment), and stems without root 
primordium (control). Three biological replicates were set for treatment and control, respectively, with a total of 12 cDNA libraries (Z2CK1, Z2CK2, Z2CK3, Z2T1, Z2T2, Z2T3, H4CK1, H4CK2, H4CK3, H4T1, H4T2 and H4T3). The transcriptome sequencing analysis yielded 38.17-50.74 million raw reads per cDNA library with a total of 528.2 million raw read for all cDNA libraries (Table 1). After removing low quality reads and reads containing adapter or poly $(\mathrm{N})$ containing, an average of $5.8 \mathrm{~Gb}$ clean data were obtained for each replicate. For all 12 cDNA libraries, the percentage of phred scores at Q20 and Q30 level exceeded $97.65 \%$ and $93.27 \%$, respectively (Table 1 ), suggesting high quality of transcript data. The remained clean reads were mapped to the Boehmeria nivea (L.) genome (Data unpublished) using HISAT (Hierarchical Indexing for Spliced Alignment of Transcripts) software. The result showed that more than $90.23 \%$ of the clean reads were mapped onto the ramie genome for all cDNA libraries, and over $63.27 \%$ were observed to be unique mapped reads (Table 1), indicating that the samples were comparable.

Table 1

Statistical analysis of transcriptome data in the 12 cDNA libraries. H4, Huazhu No4; Z2, Zhongzhu No2; T1, T2 and T3 denote the three biological replications of the treatment (cultured with tap-water); CK1, CK2 and CK3 denote the three biological replications of the control (without water treatment).

\begin{tabular}{|llllllll|}
\hline Sample & $\begin{array}{l}\text { Total } \\
\text { raw } \\
\text { read(M) }\end{array}$ & $\begin{array}{l}\text { Total } \\
\text { clean } \\
\text { read(M) }\end{array}$ & $\begin{array}{l}\text { Total } \\
\text { clean } \\
\text { base(Gb) }\end{array}$ & $\begin{array}{l}\text { Clean } \\
\text { read } \\
\text { q20(\%) }\end{array}$ & $\begin{array}{l}\text { Clean } \\
\text { read } \\
\text { q30(\%) }\end{array}$ & $\begin{array}{l}\text { Total } \\
\text { mapping } \\
\text { genome } \\
\text { ratio(\%) }\end{array}$ & $\begin{array}{l}\text { Uniquely } \\
\text { mapping } \\
\text { genome } \\
\text { ratio(\%) }\end{array}$ \\
\hline H4T1 & 41.10 & 36.03 & 5.44 & 97.78 & 93.58 & 90.51 & 63.70 \\
\hline H4T2 & 47.88 & 42.30 & 6.39 & 97.84 & 93.73 & 90.35 & 63.64 \\
\hline H4T3 & 39.33 & 34.14 & 5.15 & 97.70 & 93.39 & 90.23 & 63.27 \\
\hline H4CK1 & 45.76 & 40.17 & 6.06 & 97.81 & 93.70 & 90.37 & 65.14 \\
\hline H4CK2 & 50.74 & 44.39 & 6.70 & 97.78 & 93.59 & 90.80 & 63.98 \\
\hline H4CK3 & 45.36 & 39.87 & 6.02 & 97.80 & 93.62 & 90.67 & 64.03 \\
\hline Z2T1 & 47.07 & 40.79 & 6.16 & 97.69 & 93.37 & 90.92 & 63.61 \\
\hline Z2T2 & 44.86 & 39.44 & 5.96 & 97.81 & 93.64 & 90.47 & 65.14 \\
\hline Z2T3 & 44.13 & 38.43 & 5.80 & 97.73 & 93.47 & 91.33 & 65.72 \\
\hline Z2CK1 & 38.17 & 33.09 & 5.00 & 97.67 & 93.31 & 90.87 & 65.43 \\
\hline Z2CK2 & 43.06 & 37.19 & 5.62 & 97.65 & 93.27 & 91.00 & 65.50 \\
\hline Z2CK3 & 40.74 & 35.38 & 5.34 & 97.70 & 93.41 & 91.43 & 66.06 \\
\hline Mean & 44.02 & 38.44 & 5.80 & 97.75 & 93.51 & 90.75 & 64.60 \\
\hline Total & 528.20 & 461.22 & 69.64 & & & & \\
\hline
\end{tabular}




\section{Comparison Of Gene Expression Pattern Between Z2 And $\mathrm{H} 4$}

A total of 26723 genes including 25861 known genes and 862 novel genes were identified in the 12 cDNA libraries (Additional file 2: sheet2), with a number of 25248, 24698, 24128, and 25304 in H4CK, H4T, Z2CK, and Z2T, respectively. Most of the genes are between 300-1600 bp in length, and the number of genes whose length is more than 3000 bp was the highest, accounting for $7 \%$ of all genes. There were 22493 common genes in H4CK, H4T, Z2CK and Z2T (Fig. 2A), denoting most of the genes having similar expression behavior in the four libraries. In the treatment group (H4T vs. Z2T), there were 23940 common genes between Z2T and H4T, while there were 1364 and 758 distinctive genes in Z2T and H4T, respectively (Fig. 2B). In the control group (H4CK vs. Z2CK), the number of the distinctive genes in Z2CK and H4CK were 639 and 1759, respectively (Fig. 2C). For Z2, the number of distinctive genes in Z2T was about three times that of $\mathrm{Z2CK}$, but differently in $\mathrm{H} 4$, the number of distinctive genes in $\mathrm{H} 4 \mathrm{~T}$ was 0.58 times that of H4CK (Fig. 2D and 2E). These results may provide us with clues about why the two varieties have different rooting patterns.

\section{Identification Of Degs}

To determine the genes that were differently expressed in the two genotypes, four pairwise comparisons (H4CK vs. Z2CK, H4T vs. Z2T, H4CK vs. H4T, and Z2CK vs. Z2T) were performed. DEGs were identified by the threshold of $P_{\text {adj }}$-value $\leq 0.001$ and fold change $\geq 2$. A total of 5324, 4886, 4411, and 5195 DEGs were identified in the comparison of H4CK vs. Z2CK, H4T vs. Z2T, H4CK vs. H4T, and Z2CK vs. Z2T, respectively (Fig. 3A). In the control group (H4CK vs. Z2CK), 2074 up- and 3250 down-regulated DEGs were identified in Z2CK, while in the treatment group (H4T vs. Z2T), 3189 up- and 1697 down-regulated DEGs were found in $\mathrm{Z2T}$, suggesting different gene expression patterns in the two genotypes resulting by water treatment. In the comparisons of H4CK vs. H4T and Z2CK vs. Z2T, there were 1683 up- and 2728 down-regulated DEGs in H4, while there were 3644 up- and 1551 down-regulated DEGs in Z2, indicating that more genes were induced in $\mathrm{Z} 2$, but more genes were inhibited in $\mathrm{H} 4$. The comparisons of $\mathrm{H} 4 \mathrm{CK}$ vs. H4T and Z2CK vs. Z2T shared 1965 common genes, and there were 2446 and 3230 distinctive genes expressing in the comparison of Z2CK vs. Z2T and the comparison of H4CK vs. H4T, respectively (Fig. 3B).

\section{Go (gene Ontology) Clustering Of Degs}

GO assignment was used to classify the functions of DEGs, and significantly enriched GO term was defined when $P_{\text {adj }}$-value was less than 0.05. In total, 2879 DEGs (1146 up- and 1733 down-regulated) of $\mathrm{H} 4$ group (H4CK vs. H4T) were assigned into 43 enriched $\mathrm{GO}$ terms consisting of 17 biological process (metabolic process, cellular process, biological regulation, etc.), 14 cellular component (membrane, membrane part, cell, etc.), and 12 molecular function (catalytic activity, binding, translation regulator 
activity, etc.) (Fig. 4). Meanwhile, 3485 DEGs (2500 up- and 985 down-regulated) in Z2 group (Z2CK vs. Z2T) were assigned into 43 enriched GO terms consisting of 18 biological process (metabolic process, cellular process, pigmentation, etc.), 14 cellular component (membrane, membrane part, cell, etc.), and 11 molecular function (catalytic activity, binding, transporter activity, etc.) (Fig. 4). Eighty-nine and 106 significantly enriched $\mathrm{GO}$ terms were identified in $\mathrm{H} 4$ group and $\mathrm{Z} 2$ group, respectively (Additional file 3 :

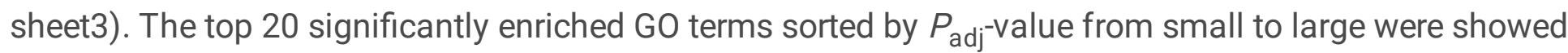
in Fig. 5. There were 11 common significantly enriched $\mathrm{GO}$ terms between $\mathrm{H} 4$ group and Z2 group, such as cell wall organization or biogenesis, oxidoreductase activity, hydrolase activity acting on glycosyl bonds, cofactor binding, and others. In each common GO term, the total number of DEGs in Z2 group was higher than that in $\mathrm{H} 4$ group, and the number of up-regulated DEGs was more than the down-regulated DEGs in $\mathrm{Z2}$ group but less in $\mathrm{H} 4$ group (Fig. 6A). Nine distinctive significantly enriched $\mathrm{GO}$ terms were found in $\mathrm{H} 4$ group which mainly included polysaccharide metabolic process, carbohydrate metabolic process, cellular carbohydrate metabolic process, cell wall macromolecule metabolic process, photosystem, and others (Fig. 6B). And the expression of the most DEGs in these distinctive GO terms were inhibited, suggesting that photosynthesis process and cell wall formation process may be suppressed in the process of root primordium formation in $\mathrm{H} 4$. Meanwhile, other nine significantly enriched GO terms were found in Z2 group, mainly including cell wall organization, integral component of membrane, membrane part, external encapsulating structure organization, and others (Fig. $6 \mathrm{C}$ ). And the number of up-regulated DEGs in these distinctive GO terms was more than that of down-regulated DEGs, indicating that cell wall formation may promote the root primordium formation in Z2.

\section{Kegg Pathway Analysis Of Degs}

To further insight the molecular interactions among the DEGs, KEGG analysis was performed and significantly enriched pathway was defined when the $P_{\text {adi }}$-value was less than 0.05 . A total of 1966 (in $\mathrm{H} 4$ group: H4CK vs. H4T) and 2381 (in Z2 group: Z2CK vs. Z2T) DEGs were enriched into 133 and 131 pathways, respectively (Additional file 4: sheet4). Thirteen and 19 significant enriched pathways were identified in the $\mathrm{H} 4$ group and the $\mathrm{Z} 2$ group, respectively. There were 6 common pathways between the two groups, while there were 7 (Photosynthesis, Photosynthesis - antenna proteins, Starch and sucrose metabolism, etc.) and 13 (MAPK signaling pathway - plant, Carotenoid biosynthesis, Plant-pathogen interaction, etc.) distinctive pathways in the $\mathrm{H} 4$ group and the $\mathrm{Z} 2$ group, respectively (Fig. 7A). In the MAPK signaling pathway of Z2 group, ethylene and jasmonic acid (JA) signaling pathways were identified (Additional file: Fig. S1).

More DEGs were induced in the Z2 group (1033 up- and 404 down-regulated), while more DEGs were reduced in the $\mathrm{H} 4$ group (424 up- and 434 down-regulated). The number of down-regulated DEGs in photosynthesis, photosynthesis - antenna proteins and amino sugar and nucleotide sugar metabolism pathways in $\mathrm{H} 4$ group were more than that of up-regulated DEGs (Fig. 7B), suggesting that photosynthesis process may be inhibited in $\mathrm{H} 4$ during the root primordium formation. In each distinct pathway of Z2 group, the number of up-regulated DEGs was more than that of down-regulated, especially 
in MAPK signaling pathway-plant, the number of up-regulated DEGs was about 2 times that of downregulated DEGs (Fig. 7C).

\section{Verification Of Expression Of Degs By Qpcr}

Four DEGs (Maker00082600, Maker00008727, Maker00075372, and Maker00063286) were randomly selected for qPCR to test the reliability of expression of DEGs in sequencing results. The results showed that there was a good agreement between the RNA-Seq data and the qPCR data (Fig. 8). After 20 days of hydroponics, the expression of Maker00082600, Maker00075372, and Maker00063286 was significantly higher than that of 10 days of hydroponics, while the expression of Maker00008727 was lower than that of 10 days of hydroponics, suggesting different expression patterns of different DEGs during root primordium formation in ramie.

\section{Discussion}

Root generation is the key factor for growth and development of asexual propagation plants [25]. Studying the molecular mechanism of rooting of ramie cuttings is helpful to improve the survival rate of ramie cuttings. However, little is known about the molecular mechanism of rooting in ramie cuttings. In this study, RNA-Seq analysis was performed to reveal the regulatory mechanism of AR in ramie cuttings using two ramie varieties ( $\mathrm{H} 4$ and $\mathrm{Z2}$ ) with different rooting patterns under the conditions of tap-water culture (treatment, $\mathrm{T}$ ) and non-water culture (control, CK). Gene expression patterns and expression of DEGs between the two varieties and within the two varieties were discussed.

In total, 26723 genes were identified in all of the detected samples, and most of the genes (22493) were common expressed in H4CK, H4T, Z2CK, and Z2T (Fig. 2A). More distinctive genes were expressed in Z2T (1799) than that in Z2CK (623), while less distinctive genes were showed in H4T (750) than that in H4CK (1300) (Fig. 2D, E), denoting different gene expression patterns in the two varieties after tap-water culture. There were more distinctive genes expressing in H4CK than that in Z2CK, but differently, there were less distinctive genes in H4T than that in Z2T (Fig. 2B, C). A total of 5324, 4886, 4411, and 5195 DEGs were identified in the comparison of H4CK vs. Z2CK, H4T vs. Z2T, H4CK vs. H4T, and Z2CK vs. Z2T, respectively (Fig. 3A). After tap-water culture, more DEGs were induced in Z2 (3644 up- and 1551 downregulated DEGs in Z2CK vs. Z2T) but more DEGs were inhibited in H4 (1683 up- and 2728 down-regulated DEGs in H4CK vs. H4T). These different expression patterns of all genes and DEGs between $\mathrm{H} 4$ and $\mathrm{Z} 2$ provide clues to the regulatory mechanism of their different rooting patterns.

There were 11 common significantly enriched GO terms between H4 group (H4CK vs. H4T) and Z2 group (Z2CK vs. Z2T), including cell wall organization or biogenesis, oxidoreductase activity, hydrolase activity acting on glycosyl bonds, cofactor binding, and others. Interestingly, in each common significantly enriched GO term, more DEGs were induced in Z2 group (2500 up- and 985 down-regulated) but more DEGs were inhibited in $\mathrm{H} 4$ group (1146 up- and 1733 down-regulated) (Fig. 6A), indicating the probable reasons of the different rooting patterns in the two varieties. Cell wall modification is an important 
biological process of root formation [26]. In H4 group, 30 DEGs related to cell wall macromolecule metabolic process were up-regulated and 8 DEGs were down-regulated, while in $\mathrm{Z2}$ group, 73 and 15 DEGs related to cell wall organization were up-regulated and down-regulated, respectively (Fig. 6B). These results suggested different molecular mechanisms of cell wall formation in the two varieties, which may result in different rooting patterns.

Photosynthesis plays an important role in root occurrence and root growth $[27,28]$. In Pinus taeda, genes related to photosynthesis are down-regulated during the root initiation phase [29]. In this study, two distinctive significantly enriched GO terms-photosystem and photosystem $\nabla$ were identified in $\mathrm{H} 4$ group, and genes involving in these $\mathrm{GO}$ terms were down-regulated, which was consistent with the previous study. In addition, KEGG analysis showed that two significantly pathways (photosynthesis and photosynthesis-antenna proteins) were distinctively identified in H4 group (Fig. 7B), and all DEGs of the two pathways were down-regulated. These results suggested that photosynthesis process was inhibited in $\mathrm{H} 4$ during the ARF, which may resulting less root primordia.

Phytohormone plays an important role in regulating rooting of cuttings [30-32]. Auxin is the primary plant hormone to promote AR in plant, and it has been found to positively regulate the occurrence and number of AR in many species [33-35]. Ethylene, salicylic acid, and brassinolide can also positively promote the occurrence and number of AR [36-38], while strigolactones, abscisic acid, gibberellins and cytokinins negatively regulate the occurrence and number of AR [39- 42]. JA has been found to synergize with auxin to promote ARF of potato stem and Petunia [43,44], but it has also been found to inhibit the occurrence of AR in Arabidopsis [45]. In this study, the MAPK signaling pathway-plant pathway was distinctively significantly identified in the $Z 2$ group, which included ethylene and jasmonic acid signaling pathways (Fig. S1). Interestingly, JA was found to interact with ethylene to regulate the occurrence and number of AR in ramie cuttings, suggesting that JA may show different regulatory mechanisms in various species.

\section{Conclusions}

Here, we provide a global view of the transcriptomic divergence of two ramie varieties with different rooting patterns under tap-water culture and non-water culture conditions using RNA-SEq. Cell wall formation, photosynthesis process and JA signaling pathway were identified to regulate ARF in ramie cuttings. Our results provide new insight into mechanisms of ARF in different ramie varieties.

\section{Abbreviarions}

AR: adventitious root; ARF: adventitious root formation; DEGs: differentially expressed genes; GO: Gene Ontology; JA: jasmonic acid; KEGG: Kyoto Encyclopedia of Genes and Genemes; qPCR: quantitative PCR.

\section{Declarations}




\section{Ethics approval and consent to participate}

Not applicable.

\section{Consent to publish}

Not applicable.

\section{Availability of data and materials}

All data presented in this study are provided either in the manuscript or additional files. Illumina sequencing data was deposited in the NCBI GEO database with accession number GSE116063.

\section{Competing interests}

The authors declare that they have no competing interests.

\section{Funding}

This work was financially supported by grants from the National Natural Science Foundation of China (31801413), the National Key Research and Development Program of China (2017YFD0800900), the Central Public-interest Scientific Institution Basal Research Fund (1610242019001), the Natural Science Foundation of Hunan Province of China (2019JJ50709), Central Public-interest Scientific Institution Basal Research Fund (1610242020001)

\section{Author Contributions}

KM.C. performed most of the experiments. B.G., P.C., JK.C. G.G. and XF.W. helped in phenotypic data and sample collection. CM.Y. helped design the experiments. AG.Z. designed the experiments. All authors read and approved the final manuscript.

\section{Acknowledgments}

We sincerely thank the key laboratory of biological and processing for bast fiber crops, ministry of agriculture of China.

\section{Supplementary information}


Additional file 1 sheet1. Primer sequences of target genes selected for qPCR.

Additional file 2 sheet2. Summary of the gene number in the 12 cDNA libraries.

Additional file 3 sheet3. Significantly enriched gene ontology (G0) terms in the pairwise comparisons of H4CK vs. H4T and Z2CK vs. Z2T.

Additional file 4 sheet4. Pathways in the comparisions of H4CK vs. H4T and Z2CK vs. Z2T identified by Kyoto Encyclopedia of Genes and Genomes (KEGG) analysis. Green parts denote significantly at Padjvalue $<0.05$

Additional file 5 Figure S1. The MAPK signaling pathway identified in the comparison of Z2CK vs. Z2T.

\section{References}

1. Vidoz ML, Loreti E, Mensuali A, Alpi A, Perata P. Hormonal interplay during adventitious root formation in flooded tomato plants. Plant J. 2010;63:551-62.

2. Calvo-Polanco M, Señorans J, Zwiazek JJ. Role of adventitious roots in water relations of tamarack (Larix laricina) seedlings exposed to flooding. BMC Plant Biol. 2012;12:99.

3. Krauss KW, Allen JA, Cahoon DR. Differential rates of vertical accretion and elevation change among aerial root types in Micronesian mangrove forests. Estuar Coast Shelf S. 2003;56:251-9.

4. Kipriotisa E, Xiong HP, Vafeiadakisc T, Kipriotia M, Alexopoulou E. Ramie and kenaf as feed crops. Ind Crop Prod. 2015;68:126-30.

5. Zhu QH, Huang DY, Liu SL, Rao ZX, Cao XL, Ren XF. Accumulation and subcellular distribution of cadmium in ramie (Boehmeria nivea L. gaud) planted on elevated soil cadmium contents. Plant Soil Environ. 2013;59:57-61.

6. Luan MB, Jian JB, Chen P, Chen JH, Chen JH, Gao Q, et al. Draft genome sequence of ramie, Boehmeria nivea (L.) Gaudich. Mol Ecol Resour. 2018;18:639.

7. Mori Y, Miyahara F, Tsutsumi Y, Kondo R. Effects of combinational treatment with ethephon and indole-3-butyric acid on adventitious rooting of Pinus thunbergii cuttings. Plant Growth Regul. 2011;63:271-8.

8. Wei K, Wang LY, Wu LY, Zhang CC, Li HL, Tan LQ, et al. Transcriptome analysis of indole-3-butyric acid-induced adventitious root formation in nodal cuttings of Camellia sinensis (L.). PLoS One. 2014;9:e107201.

9. Azad MS, Alam MJ, Mollick AS, Khan MNI. Rooting of cuttings of the wild Indian almond tree (Sterculia foetida) enhanced by the application of indole-3-butyric acid (IBA) under leafy and nonleafy conditions. Rhizosphere. 2018;5:8-15.

10. Li XP, Xu QQ, Liao WB, Ma ZJ, Xu XT, Wang M, et al. Hydrogen peroxide is involved in abscisic acidinduced adventitious rooting in cucumber (Cucumis sativus L.) under drought stress. J Plant Biol. 2016;59:536-48. 
11. Negi S, Sukumar P, Liu X, Cohen JD, Muday GK. Genetic dissection of the role of ethylene in regulating auxin-dependent lateral and adventitious root formation in tomato. Plant J. 2010;61:3-15.

12. Druege U, Franken P, Lischewski S, Ahkami AH, Zerche S, Hause B, Hajirezaei MR. Transcriptomic analysis reveals ethylene as stimulator and auxin as regulator of adventitious root formation in petunia cuttings. Frontiers Plant Sci. 2014;5:494.

13. Pacurar DI, Perrone I, Bellini C. Auxin is a central player in the hormone cross-talks that control adventitious rooting. Physiol Plantarum. 2014;151:83-96.

14. Mauriat M, Petterle A, Bellini C, Moritz T. Gibberellins inhibit adventitious rooting in hybrid aspen and Arabidopsis by affecting auxin transport. Plant J. 2014;78:372-84.

15. Baque MA, Hahn EJ, Paek KY. Growth, secondary metabolite production and antioxidant enzyme response of Morinda citrifolia adventitious root as affected by auxin and cytokinin. Plant Biotechnol Rep. 2010;4:109-16.

16. Mao J, Zhang D, Meng Y, Li K, Wang H, Han M. Inhibition of adventitious root development in apple rootstocks by cytokinin is based on its suppression of adventitious root primordia formation. Physiol Plantarum. 2019;166:663-76.

17. Rasmussen A, Mason M, De Cuyper C, Brewer PB, Herold S, Agusti J, et al. Strigolactones suppress adventitious rooting in Arabidopsis and pea. Plant Physiol. 2012;158:1976-87.

18. Sun HW, Tao JY, Hou MM, Huang SJ, Chen S, Liang ZH, et al. A strigolactone signal is required for adventitious root formation in rice. Ann Bot. 2015;115:1155-62.

19. Xu XW, Ji J, Ma XT, Xu Q, Qi XH, Chen XH. Comparative proteomic analysis provides insight into the key proteins involved in cucumber (Cucumis sativus L.) adventitious root emergence under waterlogging stress. Front Plant Sci. 2016;7:1515.

20. Li YH, Zhang HN, Wu QS, Muday GK. Transcriptional sequencing and analysis of major genes involved in the adventitious root formation of mango cotyledon segments. Planta. 2017;245:1193213.

21. Li K, Liang YQ, Xing LB, Mao JP, Liu Z, Dong F, et al. Transcriptome analysis reveals multiple hormones, wounding and sugar signaling pathways mediate adventitious root formation in apple rootstock. Int J Mol Sci. 2018;19:2201.

22. Wang LK, Feng ZX, Wang X, Wang XW, Zhang XG. DEGseq: an $R$ package for identifying differentially expressed genes from RNA-seq data. Bioinformatics. 2010;26:136-8.

23. Chen KM, Luan MB, Xiong HP, Chen P, Chen JK, Gao G, et al. Genome-wide association study discovered favorable single nucleotide polymorphisms and candidate genes associated with ramet number in ramie (Boehmeria nivea L.). BMC Plant Biol. 2018;18:345.

24. Livak KJ, Schmittgen TD. Analysis of relative gene expression data using real-time quantitative PCR and the $2^{-\triangle \Delta C T}$ method. Methods. 2001;25:402e408.

25. Carpenter WJ, Cornell JA. Auxin application duration and concentration govern rooting of hibiscus stem cuttings. J Amer Soc Hort Sci. 1992;117:68-74. 
26. Roycewicz PS, Malamy JE. Cell wall properties play an important role in the emergence of lateral root primordia from the parent root[J]. J Exp Bot. 2014;65:2057-69.

27. Smith RD, Dennison WC, Alberte RS. Role of Seagrass Photosynthesis in Root Aerobic Processes. Plant Physiol. 1984;74:1055-8.

28. Rapaka VK, Bessler B, Schreiner M, Druege U. Interplay between initial carbohydrate availability, current photosynthesis, and adventitious root formation in Pelargonium cuttings. Plant Sci. 2005;168:1547-60.

29. Brinker M. Microarray Analyses of Gene Expression during adventitious root development in Pinus contort. Plant Physiol. 2004;135:1526-39.

30. Pacurar DI, Perrone I, Bellini C. Auxin is a central player in the hormone cross-talks that control adventitious rooting. Physiol Plantarum. 2014;151:83-96.

31. Mauriat M, Petterle A, Bellini C, Moritz T. Gibberellins inhibit adventitious rooting in hybrid aspen and Arabidopsis by affecting auxin transport. Plant J. 2014;78:372-84.

32. Negishi N, Nakahama K, Urata N, Kojima M, Sakakibara H, Kawaoka A. Hormone level analysis on adventitious root formation in Eucalyptus globulus. New Forest. 2014;45:577-87.

33. Kurepin L, Haslam T, Lopez-Villalobos A, Oinam G, Yeung E. Adventitious root formation in ornamental plants: II. The role of plant growth regulators. Propag Ornam Plants. 2011;11:161-71.

34. Strader LC, Bartel B. Transport and metabolism of the endogenous auxin precursor indole-3-butyric acid. Mol Plant. 2011;4:477-86.

35. Schlicht M, Ludwig-Müller J, Burbach C, Volkmann D, Baluska F. Indole-3-butyric acid induces lateral root formation via peroxisome-derived indole-3-acetic acid and nitric oxide. New Phytol. 2013;200:473-82.

36. RÓ§nsch H, Adam G, Matschke J, Schachler G. Influence of (22S,23S)-homobrassinolide on rooting capacity and survival of adult Norway spruce cuttings. Tree Physiol. 1993;12:71-80.

37. Negi S, Sukumar P, Liu X, Cohen JD, Muday GK. Genetic dissection of the role of ethylene in regulating auxin-dependent lateral and adventitious root formation in tomato. Plant J. 2010;61:3-15.

38. Yang W, Zhu CH, Ma XL, Li GJ, Gan LJ, Ng D, Xia K. Hydrogen peroxide is a second messenger in the salicylic acid-triggered adventitious rooting process in mung bean seedlings. PLoS One. 2013;8:e84580.

39. Steffens B, Wang JX, Sauter M. Interactions between ethylene, gibberellin and abscisic acid regulate emergence and growth rate of adventitious roots in deepwater rice. Planta. 2006;223:604-12.

40. Laplaze L, Benkova E, Casimiro I, Maes L, Vanneste S, Swarup R, et al. Cytokinins act directly on lateral root founder cells to inhibit root initiation. Plant Cell. 2007;19:3889-900.

41. Lo SF, Yang SY, Chen KT, Hsing YI, Zeevaart JAD, Chen LJ, Yu SM. A novel class of gibberellin 2oxidases control semidwarfism, tillering, and root development in rice. Plant Cell. 2008;20:2603-18.

42. Guan JC, Koch KE, Suzuki M, Wu S, Latshaw S, Petruff T, et al. Diverse roles of strigolactone signaling in maize architecture and the uncoupling of a branching-specific subnetwork. Plant 
Physiol. 2012;160:1303-17.

43. Ravnikar M, Vilhar B, Gogala N. Stimulatory effects of jasmonic acid on potato stem node and protoplast culture. J Plant Growth Regul. 1992;11:29-33.

44. Lischewski S, Muchow A, Guthörl D, Hause B. Jasmonates act positively in adventitious root formation in petunia cuttings. BMC Plant Biol. 2015;15:229.

45. Gutierrez L, Mongelard G, Floková K, PÓ'curar DI, Novák O, Staswick P, et al. Auxin controls Arabidopsis adventitious root initiation by regulating jasmonic acid homeostasis. Plant Cell. 2012;24:2515-27.

\section{Figures}
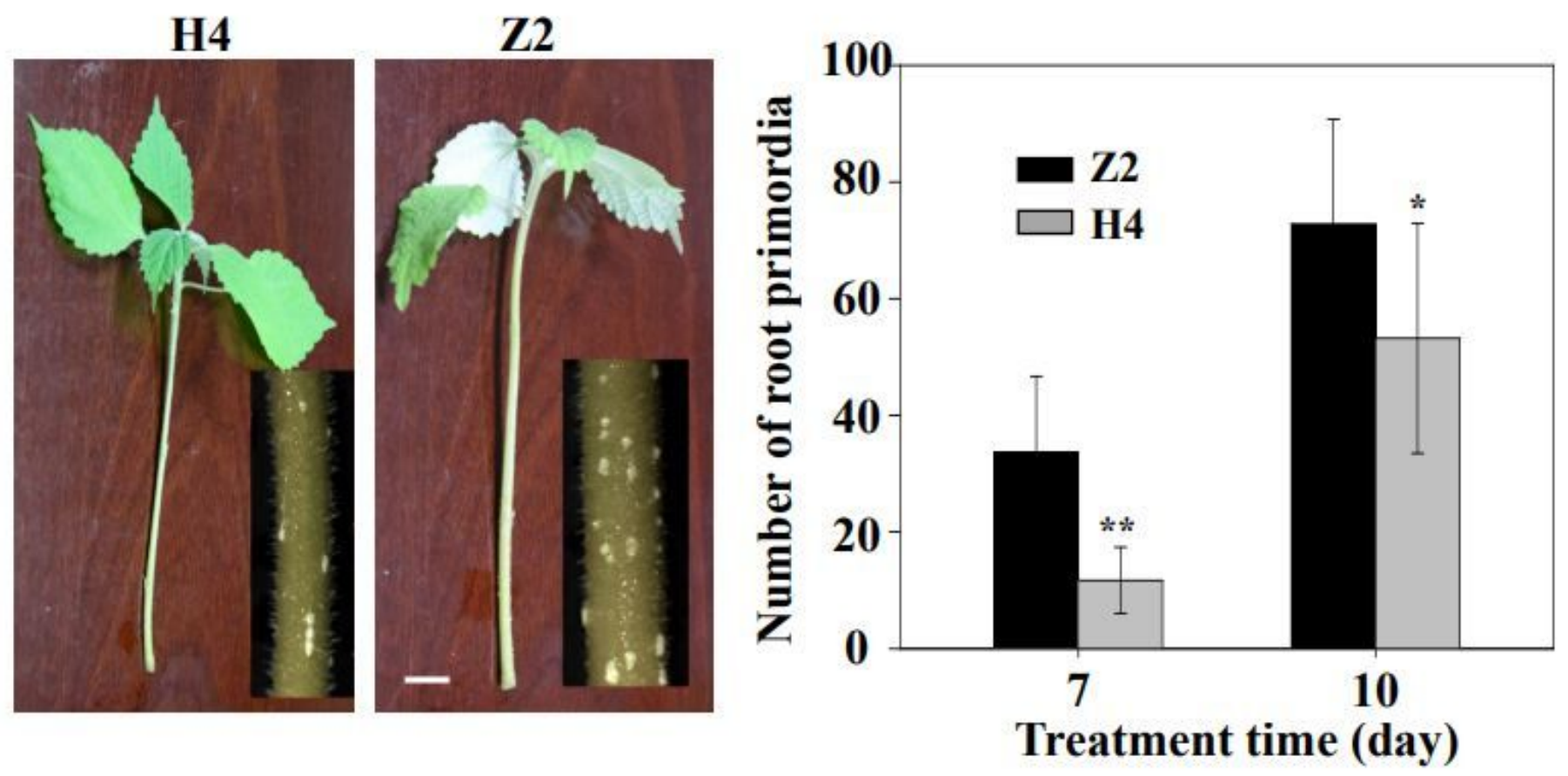

Figure 1

Phenotypic analysis of ramie cuttings cultured with tap-water. H4 and Z2 represent Huazhu No4 and Zhongzhu No2, respectively. bar $=1 \mathrm{~cm}$. The right column chart is the statistical analysis of the number of root primordia of ramie cuttings cultured with tap-water. * and ** denote significantly different from Z2 at at $P<0.05$ and $P<0.01$, respectively. Data are means $\pm S D$ of three replicates. Each replicate included five individual plants. 

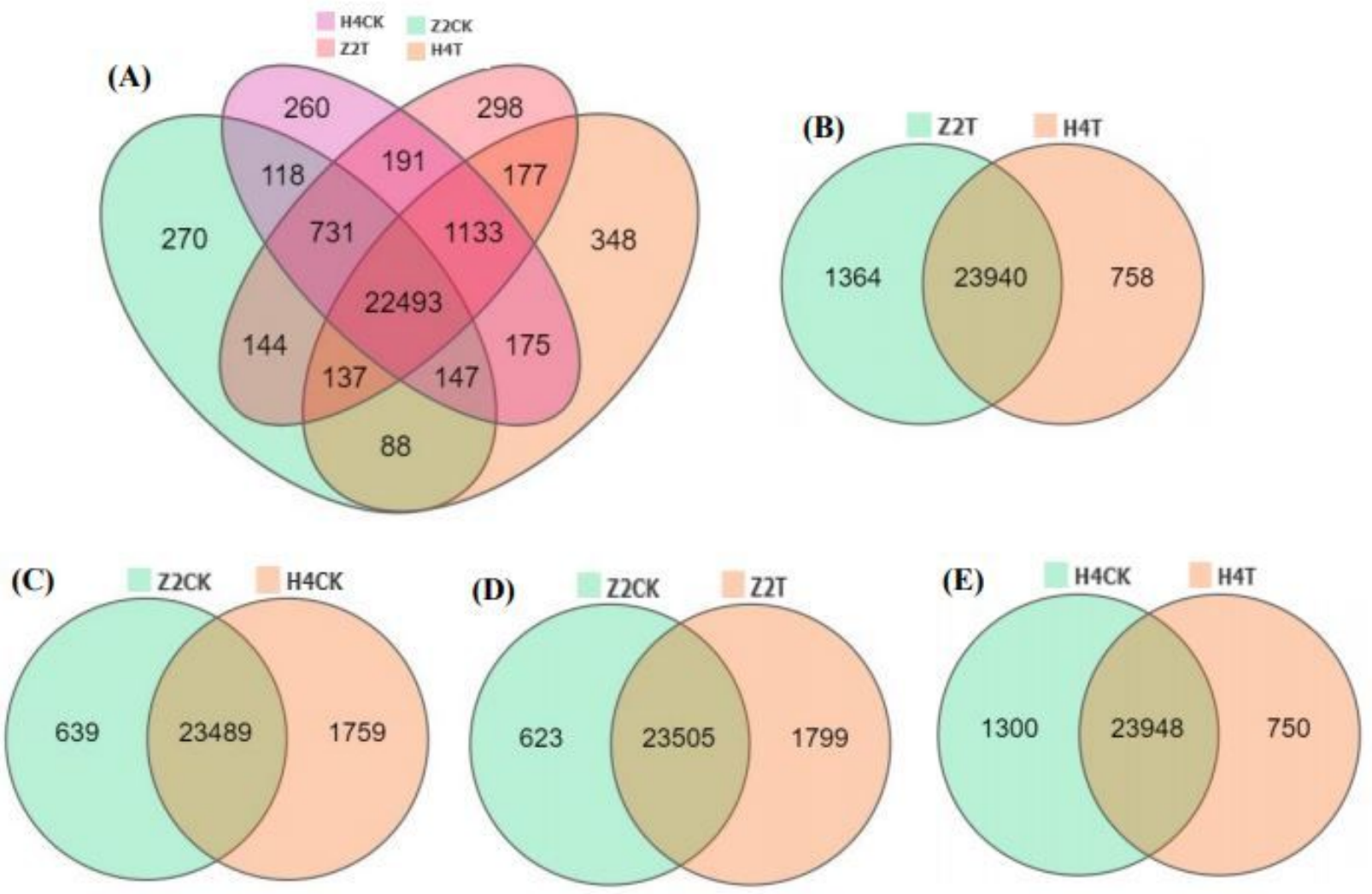

\section{Figure 2}

Venn diagram of all genes in the 12 cDNA libraries. H4CK, control of Huazhu No4; H4T, treatment of Huazhu No4; Z2CK, control of Zhongzhu No2; Z2T, control of Zhongzhu No2. 


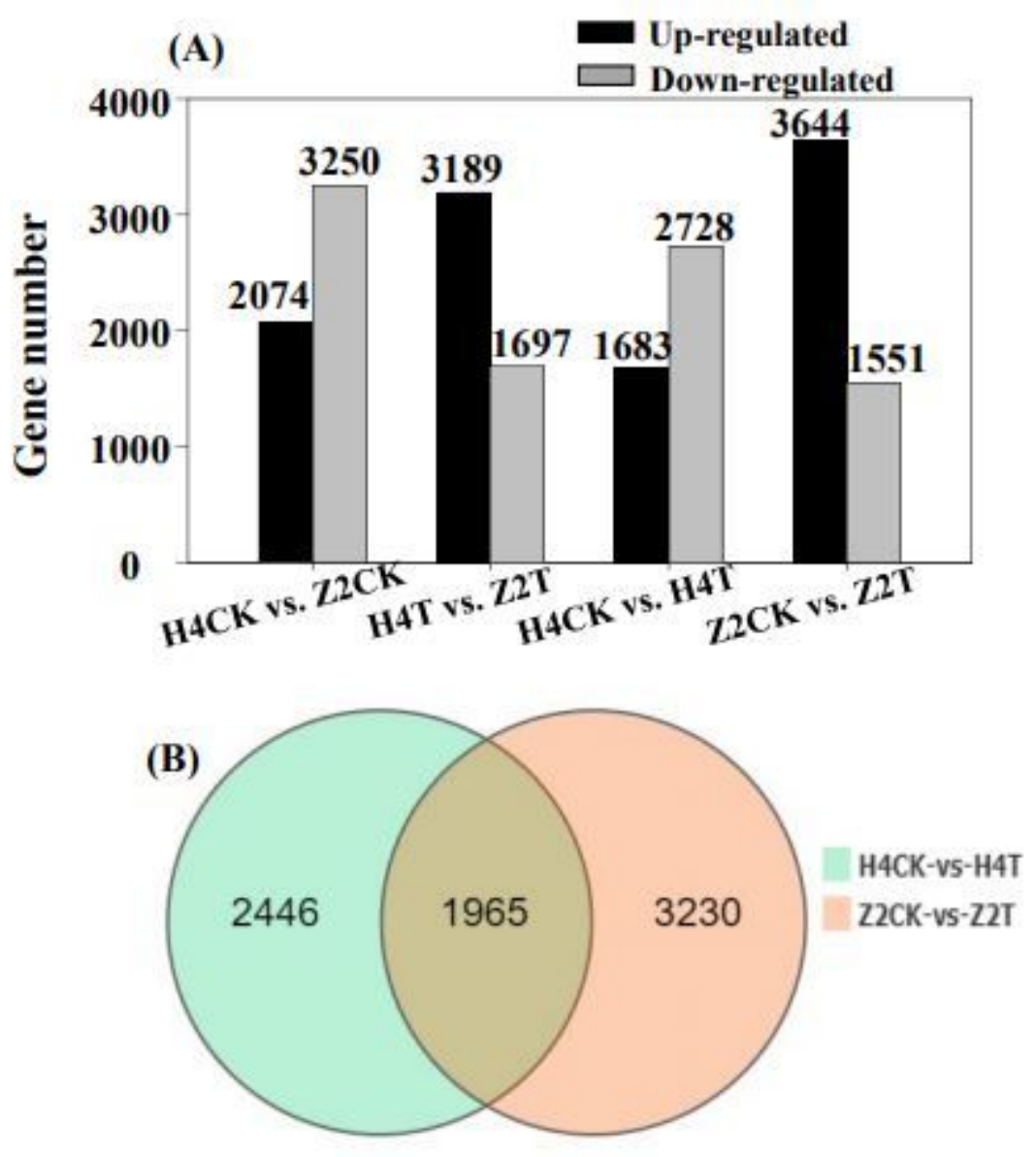

Figure 3

(A) Number of up-regulated and down-regulated differently expression genes (DEGs) in the four pairwise comparisons (H4CK vs. Z2CK, H4T vs. Z2T, H4CK vs. H4T, and Z2CK vs. Z2T). (B) Venn diagram of DEGs in the pairwise comparisons of H4CK vs. H4T and Z2CK vs. Z2T. 

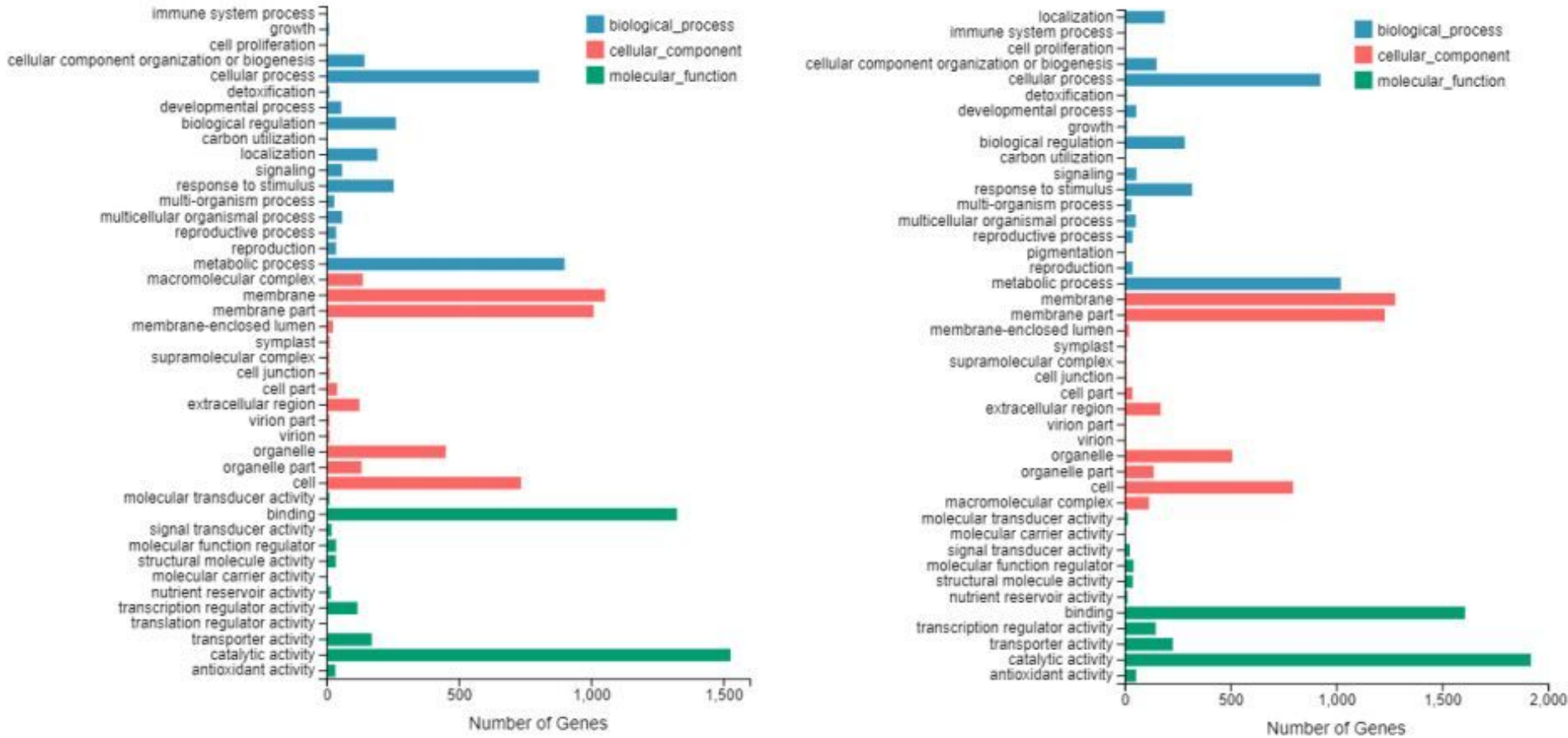

Figure 4

Gene ontology (GO) classification of DEGs in the pairwise comparisons of H4CK vs. H4T and Z2CK vs. Z2T.

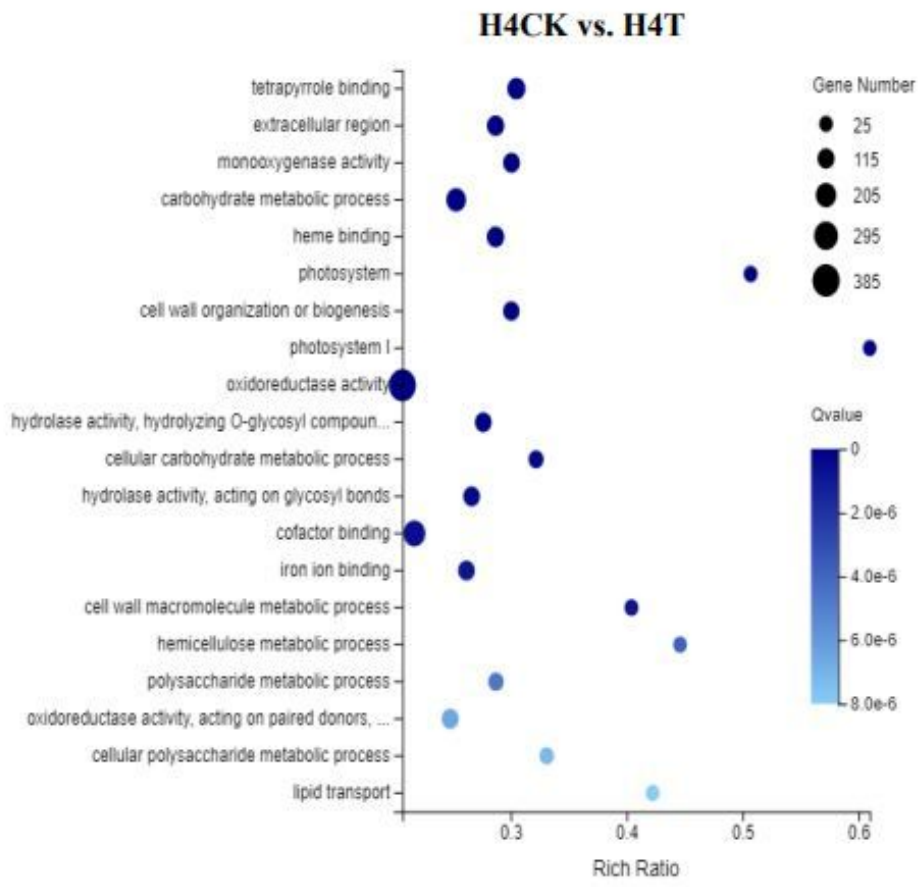

Z2CK vs. Z2T

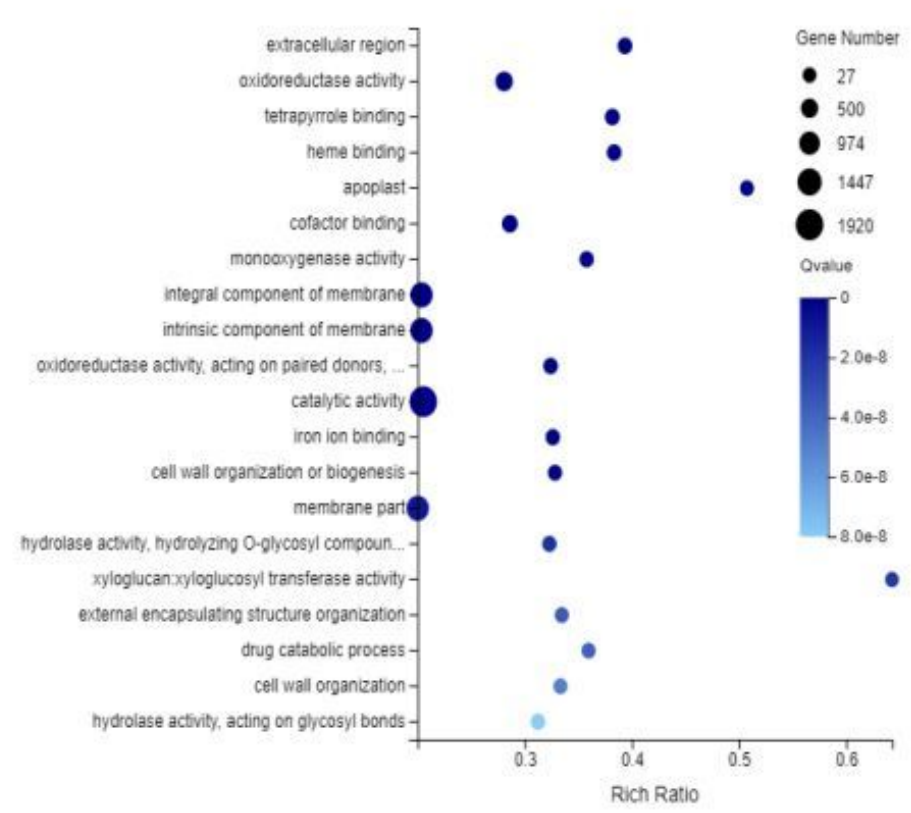

Figure 5

Gene ontology (GO) enrichment of DEGs in the pairwise comparisons of H4CK vs. H4T and Z2CK vs. Z2T. 

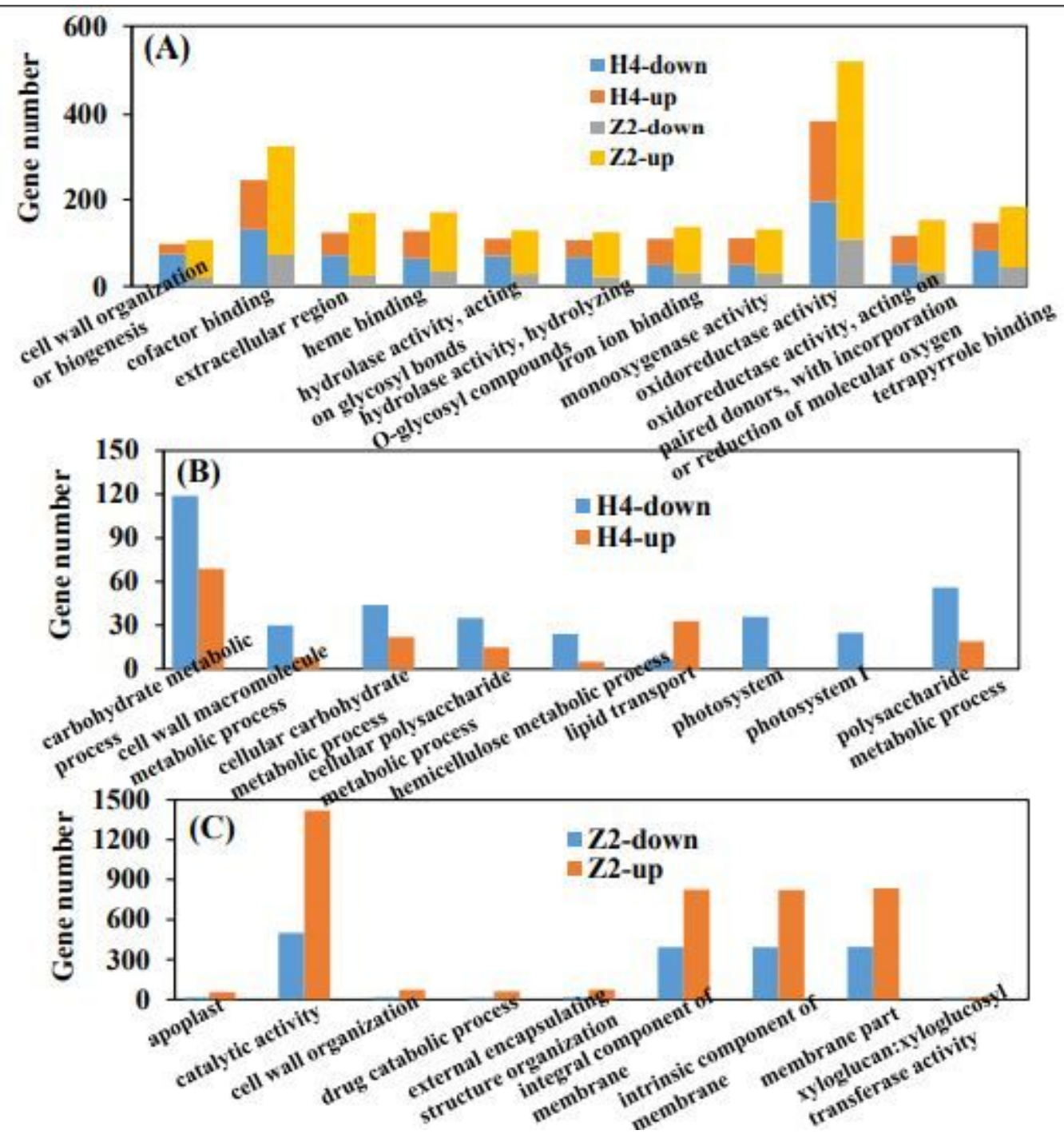

Figure 6

Statistic DEG number of each GO enriched term in the pairwise comparisons of H4CK vs. H4T and Z2CK vs. Z2T. (A) The up- and down-regulated DEG number of each common $\mathrm{GO}$ term between the comparisons of H4CK vs. H4T and Z2CK vs. Z2T. (B) The up- and down-regulated DEG number of the distinctive $\mathrm{GO}$ terms in the comparison of H4CK vs. H4T. (C) The up- and down-regulated DEG number of the distinctive GO terms in the comparison of Z2CK vs. Z2T. H4-up and H4-down denote up- and downregulated DEGs in the comparison of H4CK vs. H4T, respectively. Z2-up and Z2-down denote up- and down-regulated DEGs in the comparison of Z2CK vs. Z2T, respectively. 


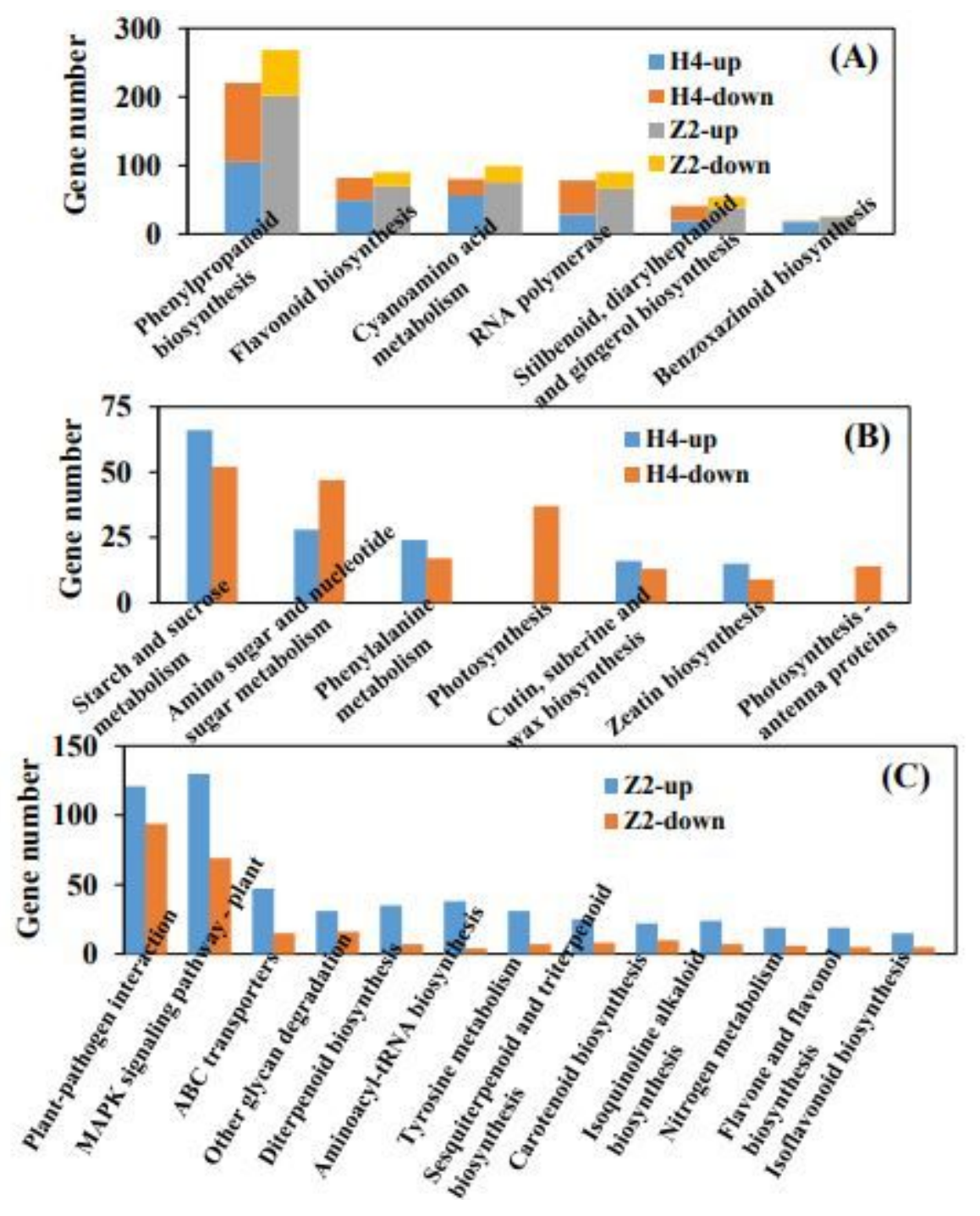

Figure 7

Statistic DEG number of each pathway identified by Kyoto Encyclopedia of Genes and Genomes (KEGG) analysis. (A) The up- and down-regulated DEG number of each common pathway between the comparisons of H4CK vs. H4T and Z2CK vs. Z2T. (B) The up- and down-regulated DEG number of the distinctive pathways in the comparison of H4CK vs. H4T. (C) The up- and down-regulated DEG number of the distinctive pathways in the comparison of Z2CK vs. Z2T. H4-up and H4-down denote up- and downregulated DEGs in the comparison of H4CK vs. H4T, respectively. Z2-up and Z2-down denote up- and down-regulated DEGs in the comparison of Z2CK vs. Z2T, respectively. 

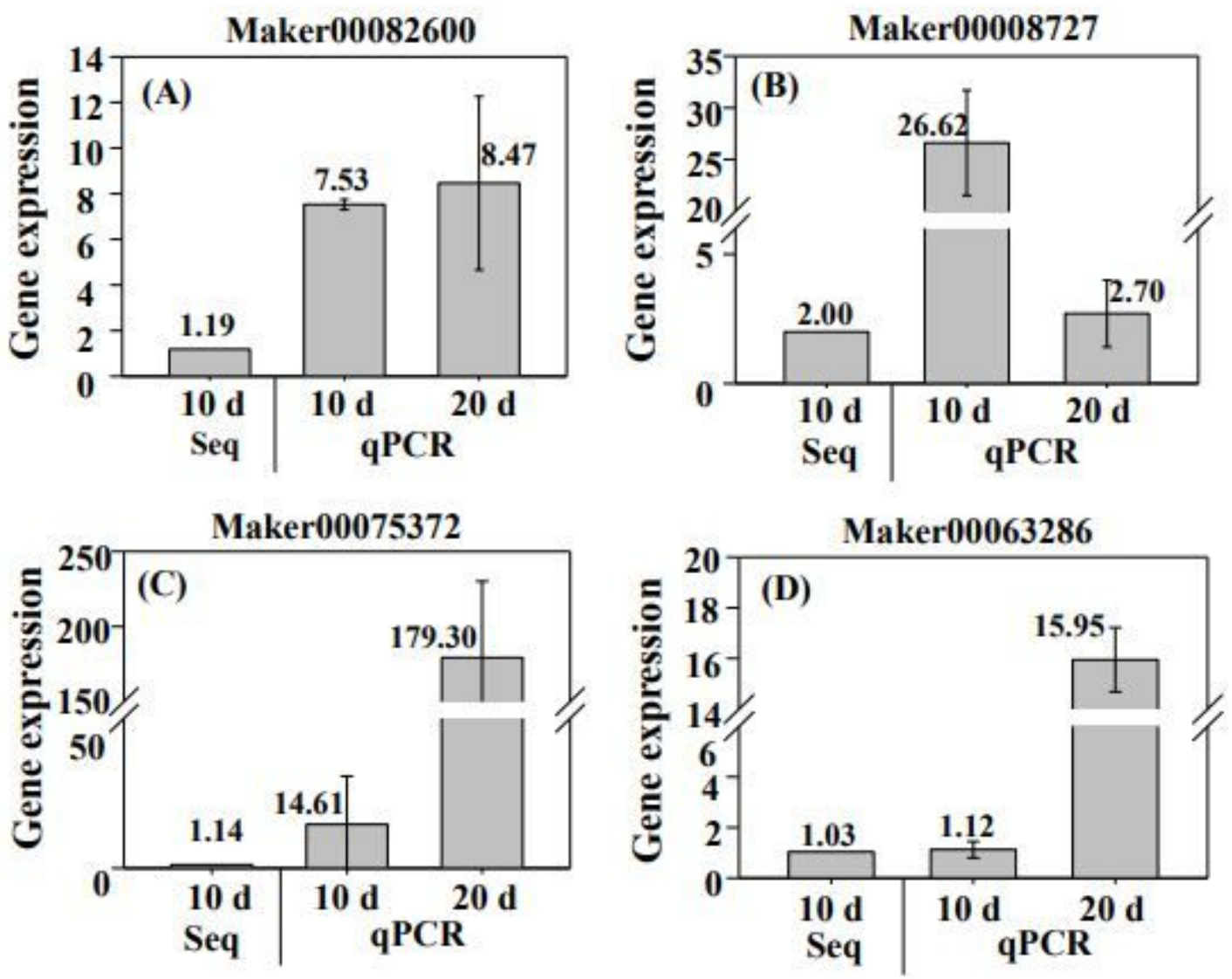

Figure 8

qPCR analysis of randomly selected DEGs in stems of ramie under tap-water cultured condition. (A) Expression of Maker00082600 in Zhongzhu No2 cultured with tap-water (Z2T). The 2- $\Delta \Delta$ Ct method was used to calculate the relative expression. The expression in Z2CK (without tap-water culture) was used as the control, and its value was set to 1. (B) Expression of Maker00008727 in Huazhu No4 cultured with tap-water (H4T). The expression in H4CK (without tap-water culture) was used as the control, and its value was set to 1. (C) and (D) Expression of Maker00075372 and Maker00063286 in Z2T. The expression in H4T was used as the control, and its value was set to 1 . For $(A)$ to $(D)$, data are means \pm SD of three replicates. "Seq" denotes the expressions obtained by RNA-Seq analysis. "qPCR" denotes the expressions obtained by qPCR.

\section{Supplementary Files}

This is a list of supplementary files associated with this preprint. Click to download.

- Additionalfile5.pdf

- Additionalfilesheets.xls 